\title{
ANÁLISE DAS VARIAÇÕES ESPACIAIS DE INDICADORES FÍSICOS E QUÍMICOS AO LONGO DO SISTEMA FLUVIAL MADEIRA/AMAZONAS
}

\author{
L. M. de CASTRO ${ }^{1}$, S. D. da ROCHA ${ }^{1}$, L. M. da $\operatorname{SILVA}^{1}$, T. C. S. de OLIVEIRA ${ }^{1}$ \\ ${ }^{1}$ Universidade Federal do Amazonas, Departamento de Química \\ E-mail para contato: lore.mady@gmail.com
}

\begin{abstract}
RESUMO - O estudo da variabilidade na concentração de substâncias ao longo de uma bacia e seus tributários permite avaliar fontes que resultam na oscilação de diferentes parâmetros. Entre as principais fontes destaca-se o aporte de elementos oriundos do carreamento de substâncias ao longo da passagem do tributário em diferentes unidades litoestratigráficas, como também influência de origem antropogênica. Este estudo consistiu na avaliação das modificações dos parâmetros: sólidos totais em suspensão, demanda química de oxigênio, turbidez, $\mathrm{pH}$ e condutividade ao longo do trecho do rio Madeira, a partir da nascente em Rurrenabaque-BO, até o rio Amazonas em Santarém-BR visando diagnosticar os possíveis subsídios que refletem na fonte de variabilidade destes parâmetros. A análise dos pontos de amostragem permitiu verificar em quais localidades os processos naturais foram suplantados pelos antropogênicos, por meio do levantamento da formação litológica e fontes potenciais de poluição da área.
\end{abstract}

\section{INTRODUÇÃO}

A Bacia do rio Madeira está localizada a sudoeste da região Amazônica onde drena uma enorme bacia hidrográfica, boa parte coberta por densa floresta tropical (Pfeiffer et al., 1991). $\mathrm{O}$ rio Madeira, que consiste em um afluente do rio Amazonas pela margem direita, possui suas nascentes na Cordilheira dos Andes e seus principais formadores são os rios Beni, Madre de Dios e Mamoré, além de possuir contribuição significativa no fluxo de sólidos carreados e na vazão para o rio Amazonas (Tucci, 2007; Bernardi et al., 2009).

Os rios estão entre os principais sistemas de transporte de matéria orgânica e inorgânica. Entre as fontes dos elementos e substâncias dissolvidas na água podem ser citadas a geoquímica do solo e das rochas, a contribuição dos componentes presentes na atmosfera e as modificações produzidas por atividades humanas (Tundisi, 2008). Queiroz et al. (2009) afirmam que alguns elementos possuem relação direta com tipos de rochas em ambientes que mantém suas características naturais e por meio desta relação é possível verificar se processos naturais foram suplantados por processos antropogênicos.

O conjunto de rios que forma a bacia do rio Madeira drena três unidades morfoestruturais principais: Planície Amazônica (44\%), Escudo Brasileiro (41\%) e cordilheira dos Andes (15\% da bacia). O trecho do rio Madeira localizado entre Porto Velho e sua foz no rio Amazonas drena rochas ígneas e metamórficas do grupo Colider, Suíte intrusiva São Romão, Suíte Intrusiva Serra da Providência, Complexo Jamari e Grupo Alto Tapajós (Figura 1) o que 
explica a heterogeneidade da composição mineralógica dos sedimentos e sólidos em suspensão desse rio influenciando diretamente composição química de suas águas (Queiroz et al., 2011).

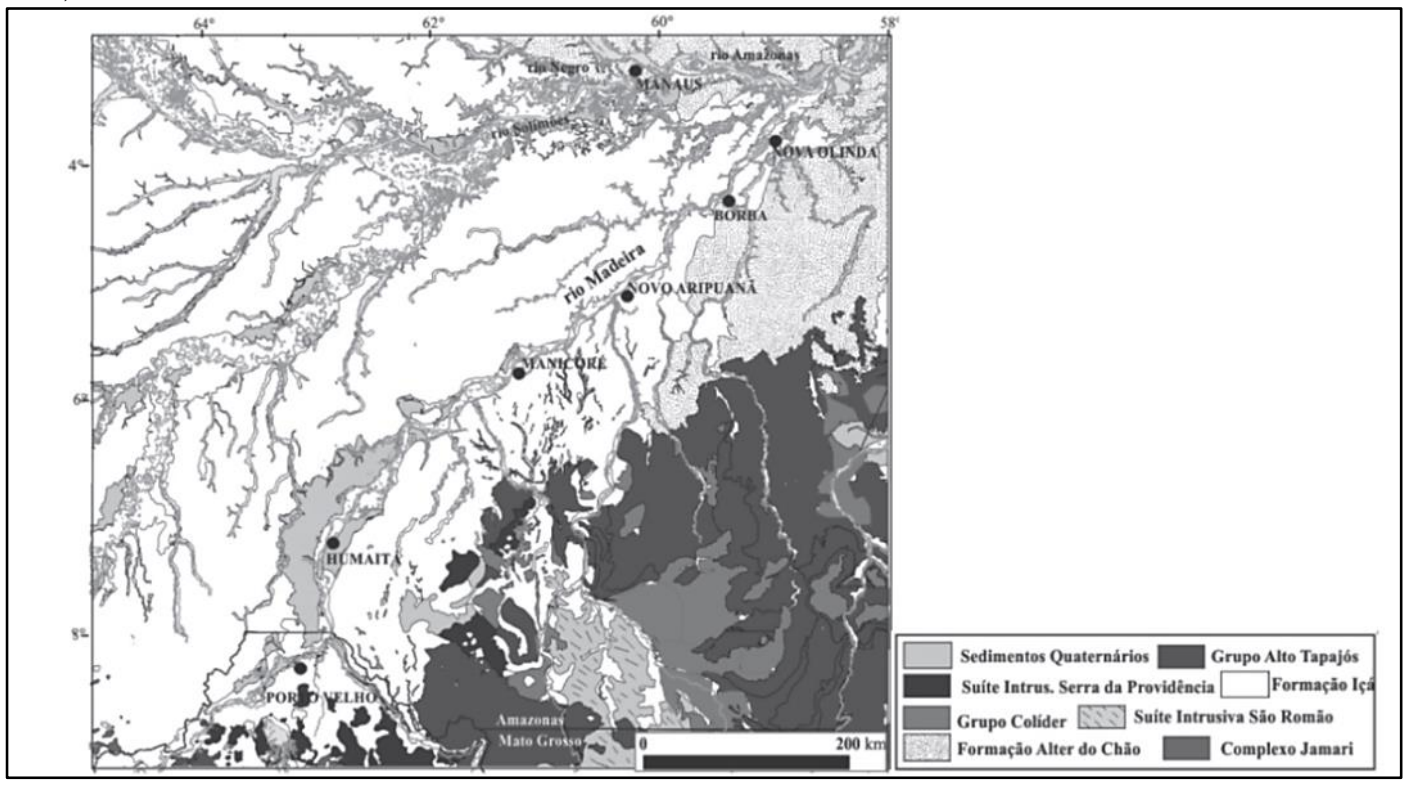

Figura 1 - Mapa geológico da região estudada (FONTE: Modificado de Queiroz et al., 2011)

Este estudo visa a análise da variação espacial de parâmetros físicos e químicos $(\mathrm{pH}$, condutividade, turbidez, demanda química de oxigênio e sólidos totais suspensos) ao longo do sistema Madeira/Amazonas por meio da verificação de valores extremos e a associação destes com as características litológicas do trecho estudado e possíveis fontes de impacto presentes ao longo do percurso do rio. O presente trabalho está inserido no escopo do projeto GEF-AM intitulado "Projeto de Gerenciamento Integrado e Sustentável dos Recursos Hídricos Transfronteiriços na Bacia do Amazonas considerando a variabilidade e mudanças climáticas" que possui o intento de diagnosticar a qualidade de ambientes aquáticos da bacia Amazônica através da análise de indicadores ambientais e a correlação destes com fontes potenciais de poluição.

\section{METODOLOGIA}

Os pontos de coleta (Figura 2) elencados estão distribuídos ao longo do curso principal do rio Madeira, afluente da margem direita do rio Amazonas, tendo início no rio Beni em Rurrenabaque - BO tendo o seu término no rio Amazonas em Santarém - BR. A seleção dos pontos se deve à proximidade destes as estações hidrométricas monitoradas pelos serviços nacionais (ORE-HYBAM) no intuito de se obter dados de vazão e cota destes rios visando a integração destes no contexto do estudo.

As campanhas de amostragem ocorreram no período de 14 a 18 de outubro de 2013 abrangendo o período de águas altas em função do regime hidrológico da estação de Óbidos BR. A estação hidrométrica de Óbidos situada no estado do Pará é considerada como sendo o ponto de referência mais a jusante que recebe as descargas dos rios Madeira, Solimões e Negro, além de que essa estação não sofre as influências da maré do Oceano Atlântico (Filizola et al., 2002) 


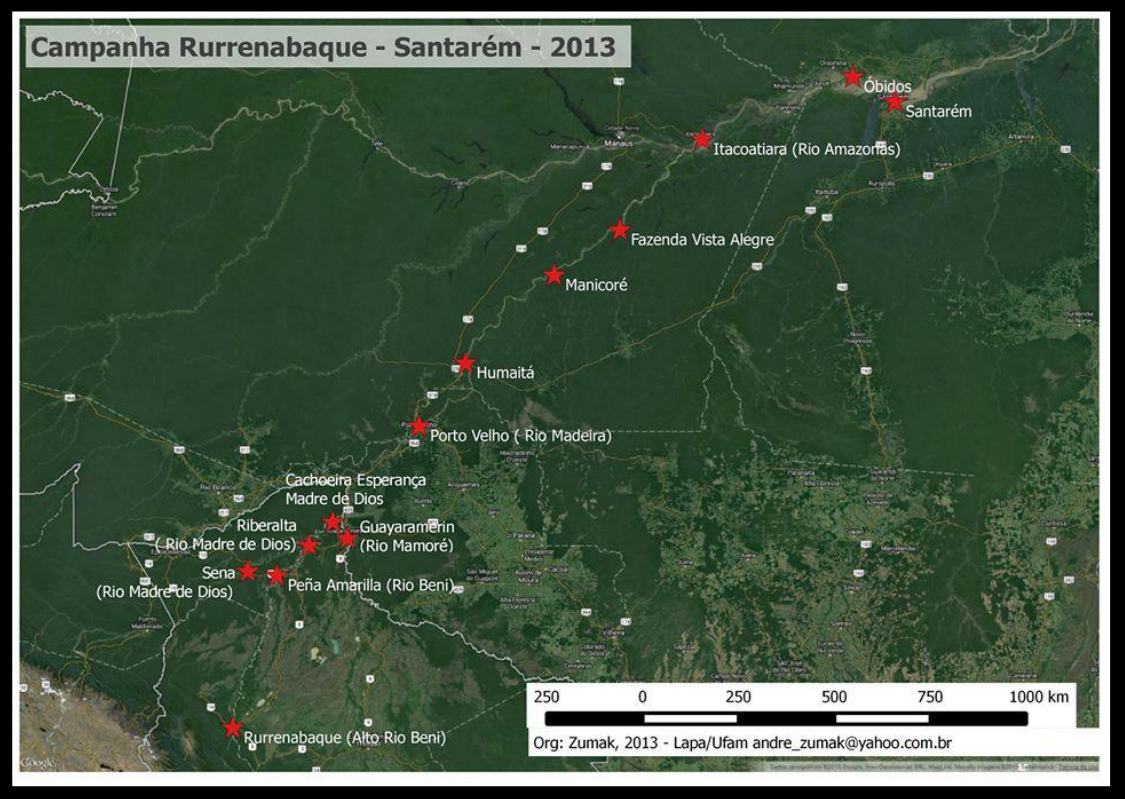

Figura 2 - Pontos de coleta elencados no sistema Madeira/Amazonas

Os pontos de coleta foram georreferenciados e listados na Tabela 1. Os parâmetros $\mathrm{pH}$, condutividade elétrica e turbidez foram mensurados in situ. No entanto, as amostras para análise dos parâmetros demanda química de oxigênio (DQO) e sólidos totais suspensos (STS) foram coletadas usando garrafa de Van Dorn por meio da imersão desta à $30 \mathrm{~cm}$ abaixo da lâmina d'água e armazenamento à temperatura de aproximadamente $4^{\circ} \mathrm{C}$ até o momento da análise. As metodologias para análise de DQO e STS foram seguidas conforme especificados por CETESB (1993) e APHA (2012), respectivamente.

Tabela 1 - Descrição dos pontos de coleta e suas respectivas coordenadas

\begin{tabular}{|c|c|c|c|c|}
\hline \multirow[t]{2}{*}{ Pontos } & \multirow[t]{2}{*}{ Rio } & \multirow[t]{2}{*}{ Município } & \multicolumn{2}{|c|}{ Coordenadas } \\
\hline & & & Longitude & Latitude \\
\hline 1 & Beni & Rurrenabaque & $65^{\circ} 35^{\prime} 5.50^{\prime \prime O}$ & $10^{\circ} 32^{\prime} 14.11 " \mathrm{~S}$ \\
\hline 2 & Madre de Dios & El Sena & $67^{\circ} 14^{\prime} 12.51 " \mathrm{O}$ & $11^{\circ} 28^{\prime} 12.60^{\prime \prime S}$ \\
\hline 3 & Beni & Peña Amarilla & $65^{\circ} 20^{\prime} 34.73^{\prime \prime O}$ & $10^{\circ} 48^{\prime} 44.71^{\prime \prime S}$ \\
\hline 4 & Beni & Riberalta & $66^{\circ} 40^{\prime} 25.70^{\prime \prime} \mathrm{O}$ & $11^{\circ} 32^{\prime} 53.68^{\prime \prime S}$ \\
\hline 5 & Beni & Cachuela Esperanza & $66^{\circ} 4^{\prime} 31.20^{\prime \prime O}$ & $10^{\circ} 59^{\prime} 40.00^{\prime \prime S}$ \\
\hline 6 & Mamoré & Guayaramerín & $67^{\circ} 32^{\prime} 6.29^{\prime \prime O}$ & $14^{\circ} 26 ' 27.49^{\prime \prime S}$ \\
\hline 7 & Madeira & Porto Velho & $63^{\circ} 47^{\prime} 29.52^{\prime \prime O}$ & $8^{\circ} 42^{\prime} 2.55^{\prime \prime S}$ \\
\hline 8 & Madeira & Humaitá & $63^{\circ} 2 ' 29.13^{\prime \prime O}$ & $7^{\circ} 31 ' 18.92 " \mathrm{~S}$ \\
\hline 9 & Madeira & Manicoré & $61^{\circ} 17^{\prime} 46.97 " \mathrm{O}$ & $5^{\circ} 44^{\prime} 56.29 " \mathrm{~S}$ \\
\hline 10 & Madeira & Fazenda Vista Alegre & $59^{\circ} 57^{\prime} 25.32^{\prime \prime O}$ & $4^{\circ} 39^{\prime} 8.22^{\prime \prime} \mathrm{S}$ \\
\hline 11 & Amazonas & Itacoatiara & $58^{\circ} 24^{\prime} 56.64^{\prime \prime} \mathrm{O}$ & $3^{\circ} 13^{\prime} 38.97^{\prime \prime S}$ \\
\hline 12 & Amazonas & Óbidos & $55^{\circ} 35^{\prime} 27.84^{\prime \prime} \mathrm{O}$ & $1^{\circ} 59 ' 26.27 " \mathrm{~S}$ \\
\hline 13 & Amazonas & Santarém & $54^{\circ} 7^{\prime} 43.84^{\prime \prime O}$ & $2^{\circ} 10^{\prime} 39.59^{\prime \prime} \mathrm{S}$ \\
\hline
\end{tabular}




\section{RESULTADOS E DISCUSSÃO}

Os valores de $\mathrm{pH}$ (Figura 3) apresentaram baixas variações no período amostrado mantendo-se próximos à neutralidade sendo atribuídos às características naturais do rio Madeira que é classificado como sendo rio de águas brancas rico em íons $\mathrm{Ca}^{2+}$ e $\mathrm{HCO}_{3}{ }^{-}$ oriundos principalmente da cordilheira dos Andes (Sioli, 1985).

Horbe \& Oliveira (2008) afirmam que a condutividade elétrica consiste na medida indireta da quantidade de íons dissolvidos presentes na água, indicando o aporte de soluções mais concentradas em elementos solúveis presentes em rochas ígneas, por exemplo. Este parâmetro pode ser influenciado por fatores tanto de origem natural como de fontes antropogênicas. Entretanto, a variação abrupta deste parâmetro pode ser indicativa de eventos de origem antrópica. A figura 3 mostra que os valores de condutividade do rio Madeira (Pontos 1 a 4) e em alguns pontos do rio Beni se mostraram acima dos demais. ANA (2011) afirma que a bacia do Madeira possui um dos maiores percentuais de uso antrópico do solo, seguida das bacias dos rios Xingu e Tapajós.

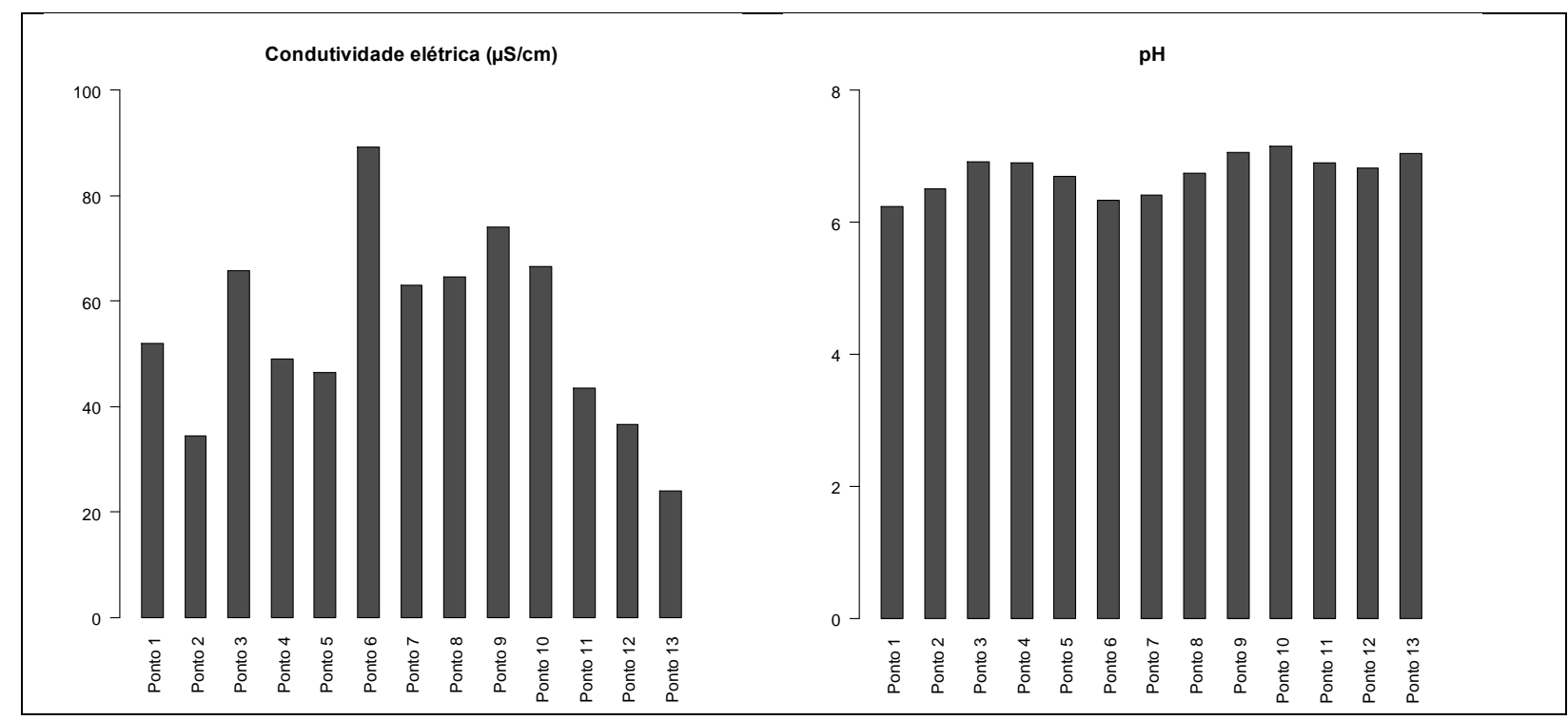

Figura 3 - Comportamento dos parâmetros condutividade elétrica $(\mu \mathrm{S} / \mathrm{cm})$ e $\mathrm{pH}$ dos pontos amostrados

O trecho do rio que drena o estado de Rondônia possui a maior extensão de registro de atividades agropecuárias (1.355.243 ha) da margem direita da bacia Amazônica, fatores estes que influenciam não somente na condutividade como também nos valores de DQO. Os maiores valores de DQO (Figura 4) foram registrados em pontos que margeiam municípios com densidade demográfica significativa, com exceção do ponto 2 (Rio Madre de Dios - El Sena), sendo que o valor elevado de DQO pode ser atribuído a influência do aporte do rio Manurimi de coloração escura, sendo esta característica geralmente atribuída à presença de produtos de decomposição de matéria orgânica. ANA (2013) relata que uma das realidades enfrentadas pelo país é baixo índice de tratamentos de esgotos em municípios de pequeno porte o que acarreta em maior aporte de matéria orgânica nos rios e consequente aumento nos valores de DQO. 


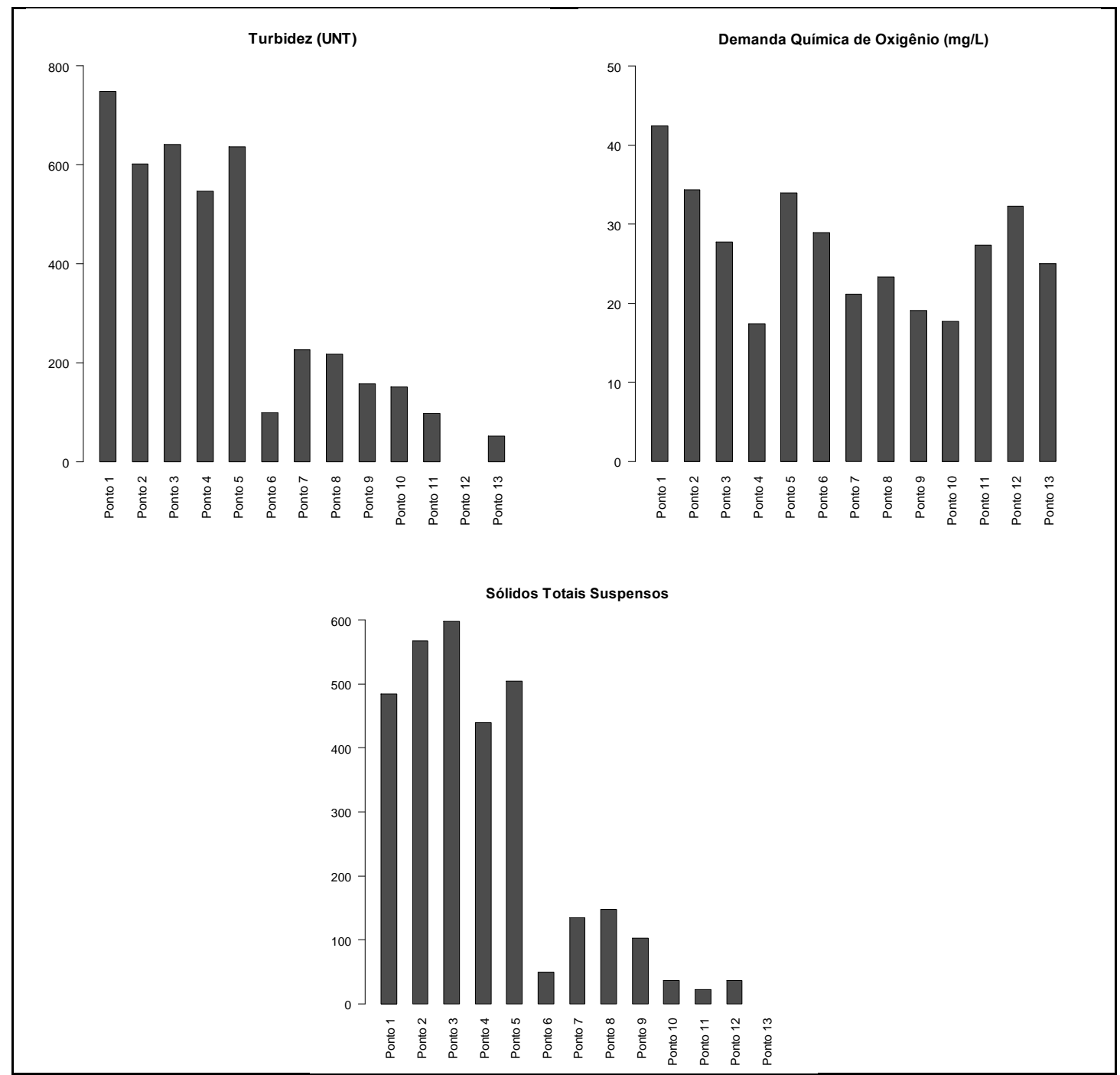

Figura 4 - Variação dos parâmetros turbidez, DQO e STS nos pontos de amostragem

Entretanto, verifica-se um decréscimo acentuado nos valores de turbidez e STS rio abaixo. Entre os fatores que podem explicar a queda abrupta destes valores destaca-se a alta sinuosidade deste rio e contínua deposição da carga material em suspensão ao longo da planície de inundação (Queiroz et al., 2011; McClain \& Naiman, 2008). Uma segunda hipótese que pode influenciar significativamente no decréscimo do material em suspensão rio abaixo é o fator de diluição. Filizola \& Guyot (2011) mensuraram a vazão média anual nos pontos de medição localizados na Fazenda Vista Alegre $\left(26.820 \mathrm{~m}^{3} \mathrm{~s}^{-1}\right)$ e em Óbidos $\left(161.100 \mathrm{~m}^{3} \cdot \mathrm{s}^{-1}\right)$, fortalecendo a hipótese que o aporte sequencial de pequenos tributários no rio principal favorece a diluição do material particulado, resultando em menores valores de turbidez e STS à jusante.

A análise da associação entre os parâmetros foi feita através da avaliação dos scores gerados em uma matriz de correlação de Spearman (não-paramétrica). Por meio desta (Tabela 2) e da matriz de p-valores (Tabela 3) é possível verificar que apenas os parâmetros turbidez e STS possuem uma alta correlação $(\sim 0,9182)$ devido ao fato deste primeiro mensurar diretamente os sólidos suspensos presentes na amostra. Destaca-se a correlação negativa entre os parâmetros pH e DQO (Score: -0,6788; p-valor: 0,02) podendo esta ser explicada devido 
aos produtos de decomposição da matéria orgânica que são constituídos predominantemente de ácidos fúlvicos e húmicos que possuem em sua cadeia grupos carboxila e hidroxila que liberam íons $\mathrm{H}^{+}$ao meio, diminuindo assim, o pH do corpo hídrico.

Tabela 2 - Matriz de correlação de Spearman para os parâmetros avaliados

\begin{tabular}{|c|c|c|c|c|c|}
\hline $\begin{array}{c}\text { Matriz de } \\
\text { Correlação: } \\
\text { Spearman }\end{array}$ & $\begin{array}{c}\text { Condutividade } \\
\text { elétrica }(\mu S / \mathrm{cm})\end{array}$ & $p H$ & $\begin{array}{c}\text { Turbidez } \\
\text { (NTU) }\end{array}$ & $D Q O$ & $S T S(m g / L)$ \\
\hline $\begin{array}{c}\text { Condutividade } \\
\text { elétrica } \\
(\mu S / \mathrm{cm})\end{array}$ & 1 & & & & \\
\hline$p H$ & 0,236902665 & 1 & & & \\
\hline $\begin{array}{c}\text { Turbidez } \\
\text { (NTU) }\end{array}$ & $-0,327272727$ & $-0,300684151$ & 1 & & \\
\hline$D Q O$ & $-0,336363636$ & $-0,678817251$ & 0,472727273 & 1 & \\
\hline$S T S(m g / L)$ & $-0,345454545$ & $-0,227791024$ & 0,918181818 & 0,481818182 & 1 \\
\hline
\end{tabular}

Tabela 3 - Matriz de p-valores para os parâmetros avaliados

\begin{tabular}{|c|c|c|c|c|c|}
\hline $\begin{array}{c}\text { Matriz de P- } \\
\text { valores }\end{array}$ & $\begin{array}{c}\text { Condutividade } \\
\text { elétrica } \\
(\mu \mathrm{S} / \mathrm{cm}) \\
\end{array}$ & $p H$ & $\begin{array}{l}\text { Turbidez } \\
\text { (NTU) }\end{array}$ & $D Q O$ & $S T S(\mathrm{mg} / \mathrm{L})$ \\
\hline $\begin{array}{l}\text { Condutividade } \\
\text { elétrica }(\mu \mathrm{S} / \mathrm{cm})\end{array}$ & 1 & & & & \\
\hline$p H$ & 0,483065408 & 1 & & & \\
\hline Turbidez (NTU) & 0,327015951 & 0,368938991 & 1 & & \\
\hline$D Q O$ & 0,313026083 & 0,021636578 & 0,141371244 & 1 & \\
\hline$S T S(m g / L)$ & 0,299387684 & 0,500533831 & 0 & 0,133149576 & 1 \\
\hline
\end{tabular}

Apesar dessa propriedade ser melhor visualizada em rios de águas pretas, parte da acidez das águas dos rios de coloração branca se deve a presença dessas substâncias orgânicas que são provenientes da drenagem de solos arenosos que são cobertos por diversos tipos de vegetação como: caatingas amazônicas, campina ou campinarana (Oliveira et al., 2001).

\section{CONSIDERAÇÕES FINAIS}

A heterogeneidade dos ambientes drenados pelo rio Madeira dificulta a distinção dos fatores que influenciam na dimensão dos parâmetros avaliados. No entanto, é possível verificar que apesar da incidência notável de fontes de origem antropogênica em alguns trechos do rio, principalmente no estado de Rondônia e em pontos localizados à beira de municípios com densidade demográfica significativa, o rio Madeira devido à sua larga extensão possui uma dinâmica que promove a sua autodepuração, resultando na manutenção de suas características naturais. O gerenciamento à luz do Planejamento Integrado de Recursos de bacias hidrográficas permite dimensionar as principais influências que mudanças 
no uso e cobertura do solo possam estar exercendo na qualidade dos grandes rios e seus tributários.

\section{REFERÊNCIAS BIBLIOGRÁFICAS}

AGÊNCIA NACIONAL DE ÁGUAS (ANA); Plano estratégico de recursos hídricos dos afluentes da margem direita do rio Amazonas. Vol. 1, 2011.

AGÊNCIA NACIONAL DE ÁGUAS (ANA); Conjuntura dos recursos hídricos no Brasil. Brasília: ANA, 432p., 2013.

APHA - American Public Health Association; American Water Work Association AWWA; Water Pollution Control Federation - WPCF. Standard Methods for the Examination of Water and Wastewater. 22 ed. New York, 1268p, 2012.

BERNARDI, J. V. E.; LACERDA, L.D.; DÓREA, J.G.; LANDIM, P.M.B.; GOMES, J. P. O.; ALMEIDA, R.; MANZATTO, A.G.; BASTOS, W.R.; Aplicação da análise das componentes principais na ordenação dos parâmetros físico-químicos no alto rio Madeira e afluentes, Amazônia Ocidental. Geoc. Bras., Vol. 23, n. 1, p. 001-158, 2009.

COMPANHIA AMBIENTAL DO ESTADO DE SÃO PAULO - CETESB; Determinação de oxigênio consumido em águas - método do permanganato de potássio: método de ensaio L5.143, São Paulo: CETESB, 1993.

FILIZOLA, N.; GUYOT, J. L.; MOLINIER, M.; GUIMARÃES, V.; OLIVEIRA, E.; FREITAS, M. A.; Caracterização hidrológica da Bacia Amazônica. Amazônia uma perspectiva interdisciplinar. EDUA. p.33-53. Manaus, 2002.

FILIZOLA, N.; GUYOT, J. L. Fluxo de sedimentos em suspensão nos rios da Amazônia. Revista Brasileira de Geociências, v. 41, p. 566-576, 2011.

HORBE, A. M. C.; SOUZA, L. G. Química de igarapés de água preta do nordeste do Amazonas - Brasil. Acta Amaz., Vol.38 (4), p. 753-760, 2008.

MCCLAIN, M. E.; NAIMAN, R. J.; Andean influences on the biogeochemistry and ecology of the Amazon River. Bioscience, Vol. 58, p. 325 a 338, n. 4, 2008.

OLIVEIRA, Fernando J. S. O. Biorremediação de solo arenoso contaminado por óleo cru. Dissertação de mestrado. Universidade Federal do Rio de Janeiro, 2001.

PFEIFFER, W. C.; MALM, O; SOUZA, C. M. M.; DRUDE DE LACERDA, L.; SILVEIRA, E. G.; BASTOS, W. R.; Mercury in the Madeira River ecosystem, Rondônia, Brazil. Forest Ecol. and Manag.. Vol. 38, p. 239 a 245, 1991.

QUEIROZ, M. M. A.; HORBE, A. M. C.; SEYLER,P.; MOURA, C.A.V. Hidroquímica do rio Solimões na região entre Manacapuru e Alvarães - Amazonas- Brasil. Acta Amaz. Vol. 39, n. 4, p. 943 à 952, 2009.

QUEIROZ, M. M .A.; HORBE, A. M. C.; SEYLER,P.; MOURA, C.A.V. Mineralogia e química dos sedimentos de fundo do médio e baixo Madeira e de seus principais tributários Amazonas - Brasil. Acta Amaz., Vol. 41, n. 4, p. 453 à 464, 2011.

SIOLI, H. Amazônia - Fundamentos da Ecologia da maior região de Florestas Tropicais. Trad. J. Becker. Rio de Janeiro: Vozes, 72p, 1985.

TUCCI, C. E. M.; Análise dos estudos ambientais dos empreendimentos do rio Madeira. Instituto Brasileiro de Meio Ambiente - IBAMA, 2007.

TUNDISI, J. G.; TUNDISI, T. M.; Limnologia. São Paulo, Oficina de textos, 631 p., 2008. 